\section{Royal Institution is ever more relevant}

You mischaracterize the impact and continued relevance of the

Royal Institution of Great Britain (RI) by presenting an incomplete picture (Nature 493, 452; 2013).

In 2012 the RI delivered 87 evening events. Of the 46 held in the Faraday Theatre, the mean attendance was 288 , much higher than might be expected from a small marketing budget. The thriving schools programme featured 136 lectures and workshops, reaching nearly 13,000 students last year alone. The RI runs mathematics and engineering masterclasses for schoolchildren at more than 140 UK locations. Our activities score very highly using the industry-standard Generic Learning Outcomes, which gauge enjoyment, inspiration, knowledge and understanding.

Thanks to its unique position and unrivalled heritage, the RI attracts the best scientists and science communicators across its programmes, including psychologist Stephen Pinker and physicist Brian Cox.

Even if one thinks that public talks are irrelevant in this age of "the Internet and mass media", then the RI is still a powerful player. Our televised Christmas Lectures had an audience of 4.2 million in 2011.

The RI Channel website launched just over a year ago and showcases some 300 videos, which have so far attracted almost 1 million views. Some highlight recent RI events, others feature re-digitized footage from our archive, and there are high-quality videos from scientific institutions across the world.

I accept that mistakes made by the RI have led to the current situation. The growing popularity of its programmes live, broadcast and online - isn't one of them.

Gail Cardew Royal Institution of Great Britain, London.

gail@ri.ac.uk
See go.nature.com/12ymkg for more debate on this topic.

\section{Sometimes Bayesian statistics are better}

David Vaux argues that experimental biologists should be better versed in classical statistics (Nature 492, 180-181; 2012). We suggest that they might also join the shift to Bayesian statistics that is already under way in many other areas of science.

He defines the $95 \%$ confidence interval (CI) as "with 95\% confidence, the population mean will lie in this interval", adding that it is commonly used "to infer where the population mean lies, and to compare two populations".

However, a 95\% CI merely tells us that if we were to sample from the population many times and calculate a $95 \% \mathrm{CI}$ for each sample, $95 \%$ of the calculated CIs would, on average, contain the true population mean. Because classical statistics concern conditional probabilities of data based on assumed true parameter values (namely, the plausibility of the observed or more extreme data, given our assumptions), the $95 \%$ CI does not allow a probabilistic inference about "where the population mean lies".

Bayesian statistics, by contrast, provide conditional probabilities of parameter values - the plausibility of different parameter values - given the data. Bayesian statistics therefore allow for probabilistic inferences about the true population mean and other parameters (J. K. Kruschke J. Exp. Psychol. Gen. http://doi. org/kdb; 2012).

Researchers often confuse probabilities derived from classical statistics ( $P$ values, for example) with Bayesian posterior probabilities (G. Gigerenzer J. Socio-Econ. 33, 587-606; 2004). This is because the latter represent what scientists are ultimately interested in: the conditional probability of parameter values or hypotheses, given the data. Stefan Herzog, Dirk Ostwald
Max Planck Institute for Human Development, Berlin, Germany. herzog@mpib-berlin.mpg.de

David Vaux replies: I agree that it is often preferable to use Bayesian rather than classical statistics, and that I did not give the full and precise definition of $95 \%$ confidence intervals in my Comment. However, the distinction is unimportant for experiments in which $N$ is 3 or less (where $N$ is the number of independent samples).

Cell and molecular biologists could learn from physicist Ernest Rutherford, who said: "If your experiment needs statistics, you ought to have done a better experiment." Where $N$ is small, they would do well to plot all data points, rather than showing any statistics, classical or Bayesian.

\section{Species splitting puts conservation at risk}

A worrying trend over the past decade has been the taxonomic splitting of mammal species, mostly by raising subspecies to species. Because of its potential bearing on conservation, we advise caution in this practice, which we maintain should be based solely on peer-reviewed evidence of biological validity.

This trend is mainly the result of a shift from the biological to the phylogenetic species concept. The biological species concept holds that species are groups of (actually or potentially) interbreeding populations. The phylogenetic species concept and its variants, by contrast, define species either as the smallest cluster sharing genetically transmitted characters, such that all individuals are unequivocally diagnosable on the basis of those characters, or as monophyletic assemblages. In these, all individuals sharing a common ancestor belong to one species, with common ancestry inferred on the basis of shared derived characters (see, for example, C. Groves and P. Grubb Ungulate
Taxonomy Johns Hopkins University Press, 2011).

As well as confusing the functional meaning of a species, taxon splitting could be detrimental to conservation. If threatened species are incorrectly split into several units and managed as such, for example in captive breeding or metapopulation management, there could be unnecessary loss of genetic variation and an increased risk of extinction.

Such newly designated species call into question the suitability of Red List assessments and the legality of species identified under national laws and international agreements. It is vital to identify true species as conservation units, based on adequate sample sizes and on information pertaining to genetics, morphology and behaviour.

Frank E. Zachos ${ }^{\star}$ Natural History Museum Vienna, Austria. frank.zachos@nhm-wien.ac.at ${ }^{\star}$ On behalf of 6 co-signatories (see go.nature.com/4urduh for full list).

\section{Another collider is not the way forward}

You encourage the international particle-physics community to support Japan's offer to host the next big particle collider, the International Linear Collider (ILC; Nature 492, 312; 2012). But the case for the ILC is not strong at this point, considering that the Large Hadron Collider (LHC) in Switzerland has revealed only one standard-model Higgs-bosonlike particle so far.

The European strategy for particle physics will shortly be updated, but many European countries are hesitant to invest taxpayers' money in another largescale collider (Nature Physics 9, 1;2013). Instead, the community should be focusing on the LHC, its future upgrades and other, smaller-scale physics projects. Tommy Ohlsson $K T H$ Royal Institute of Technology, Stockholm, Sweden. tohlsson@kth.se 